\title{
BRONCHIECTASIS IN CHILDREN: ITS MULTIPLE CLINICAL AND PATHOLOGICAL ASPECTS
}

\author{
BY \\ HOWARD WILLIAMS and R. N. O'REILLY \\ From the Clinical Research Unit, Royal Children's Hospital, Melbourne
}

(RECEIVED FOR PUBLICATION DECEMBER 12, 1958)

There has been considerable controversy concerning the aetiology, pathology and pathogenesis of bronchiectasis ever since Laennec's original description in 1819. The two principal and most widely accepted theories of pathogenesis have been first, that the disease was primarily due to bronchial damage from infection, the weakened bronchi then dilating, and second, that pulmonary collapse was the primary cause, bronchial dilatation initially being due mainly to secondary mechanical factors. Pathological studies have revealed a wide variety of lesions. In some the essential structure of the bronchi and bronchioles was completely destroyed by infection, in others the component parts of the bronchial wall were normal. Collapse of the affected lobes or segments was a feature in some, but not in others. Interstitial inflammation of the adjacent lung could be either pronounced or absent. Clinical studies have also revealed great diversity of features in different patients. The mode of onset may vary from an insidious onset with bronchitis in infancy to an acute onset with either severe pneumonia or pulmonary collapse. Some patients have no symptoms and live a normal life, others have much cough and sputum and die early from pulmonary suppuration. Some have chronic sinus infection and wax keratosis, others never show these features.

What is the explanation of this diversity of clinical and pathological features, and theories of pathogenesis? Two explanations are possible: first, there are all grades of severity of a disease entity which may be caused by a variety of infections, and second, there are a number of separate diseases each with a different pathology and clinical pattern but all with the one common gross morphological feature of bronchial dilatation. This problem has been considerably elucidated in recent years by detailed clinical and pathological studies. It is now well known that primary pulmonary tuberculosis
(Kent, 1942; Jones, Peck and Willis, 1946; Veeneklaas, 1952; and Williams and Anderson, 1953) and fibrocystic disease of the pancreas (Zuelzer and Newton, 1949; Bodian, 1952; Sant' Agnese, 1955) give rise to bronchiectasis, and that both diseases have distinctive pathological and clinical features. Whitwell (1952) in a morbid anatomical study of bronchiectatic lobes resected surgically described three separate pathological entities, atelectatic, saccular and follicular bronchiectasis and indicated that there were distinctive clinical features in each group. He was unable to classify a considerable proportion of his material.

In a study of the development of bronchiectatic lesions in a wide variety of patients, we have been able to classify them into a number of definite groups according to their primary pathology. In a series of 650 patients with bronchiectatic lesions we have studied 241 of them from the inception of the disease process and followed them for periods of four to 10 years. The following classification is submitted but it is proposed to give details of the first two groups only, those with subacute pyogenic collapse and those with non-specific infective bronchiolitis and interstitial pneumonia, as the pathology and clinical features of the other groups are better known and understood.

(1) Sub-acute pyogenic pulmonary collapse ..

(2) Non-specific infective bronchiolitis and/or

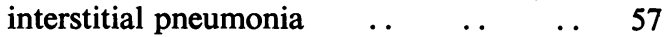

(3) Fibrocystic disease of the pancreas $\quad$. 56

(4) Primary pulmonary tuberculosis .. $\quad$.. 53

(5) Congenital malformations of the bronchial

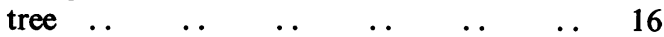

(6) Miscellaneous lung infections $\quad \ldots \quad \ldots \quad 22$ 
Subacute Pyogenic Pulmonary Collapse

Permanent and Reversible Bronchiectatic Changes. The common association of bronchiectasis and collapse both clinically and pathologically in humans, and experimentally in animals led to the assumption that pulmonary collapse was the major factor causing bronchiectasis. Warner and Graham (1933), Anspach (1934). Lee Lander and Davidson (1938), Fleischner (1940), Brennemann (1943), Lee Lander (1946) and Coope (1948) have all expressed this view. It has been also demonstrated that bronchial dilatation in a collapsed lobe may disappear if the lobe re-expands, by Findlay (1935), Jennings (1937), Lee Lander (1946) and Field (1949).

Material and Methods. Subacute pyogenic pulmonary collapse has been arbitrarily defined as a pyogenically infected collapsed lobe or segment which has been collapsed between six weeks and one year. After one year it was considered to be chronic. Collapse associated with fibrocystic disease of the pancreas or tuberculous glands was excluded in these patients. Of the $\mathbf{3 7}$ children in the group, non-specific respiratory infection was the cause of collapse in 16, pertussis in 11, measles in two, allergic bronchitis in four, foreign body in two and post-operative causes in two. All the patients were studied clinically, special note being made of previous general health and respiratory disease. The follow-up period has varied from four to 10 years. Special methods of study were serial anteroposterior and lateral chest radiographs, bronchoscopy and bronchogram. The sputum was cultured for pathogenic organisms using standard $6 \%$ horse blood agar plates with a meat infusion broth base. Special techniques for culture of Haemophilus influenzae were not used routinely.

Age, Sex Incidence, Predisposing Factors. All different age groups were represented, four children being under 2 years, 20 between 2 and 6 years and 13 between 6 and 14 years. Eighteen of these were boys and 19 girls. . All except five children had been in previous good health. Four of the five had previous manifestations of an allergic diathesis, while the fifth had suffered from recurrent respiratory infections. An uncle of one patient had bronchiectasis.
Onset and Symptoms. In all patients the onset of the illness was a clearly defined episode with either acute respiratory infection, pertussis, morbilli, an attack of allergic bronchitis, a post-operative complication or inhalation of a foreign body. The two outstanding clinical features were, first, persistent cough following the initial respiratory infection, and second, constitutional symptoms of ill health. There were no characteristic features about the cough, although some parents described it as 'chesty' or as if 'phlegm were present', but no child spontaneously expectorated sputum. The constitutional symptoms were those common to any low grade infection, namely pallor, languor, poor appetite, loss of weight and often continuous or intermittent low grade fever.

Clinical Signs. The patients did not look well, their muscle tone and posture was poor and all except three had lost weight. Three had upper respiratory tract infection. Physical signs in the chest were localized to the affected lobe or lobes and usually minimal. Diminished air entry and crepitations were characteristic of the majority, but tubular breathing was heard in four. Postural coughing over the examiner's knee (Fig. 1) and urging the child to 'cough and spit it up' was frequently successful in demonstrating the presence of mucopus in the bronchial tree.

Radiological signs of lobar collapse were un-

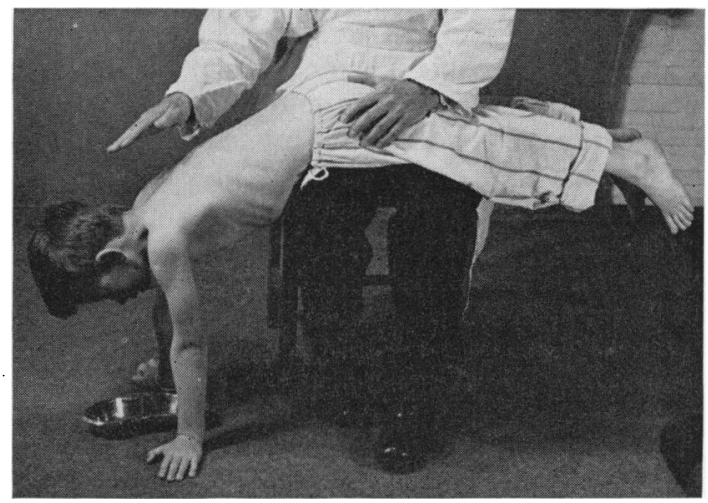

FIG. 1.-Showing method of posturing child to obtain sputum.

TABLE 1

LOBAR DISTRIBUTION

\begin{tabular}{|c|c|c|c|c|c|c|c|c|c|}
\hline \multicolumn{5}{|c|}{ Unilateral } & \multicolumn{5}{|c|}{ Bilateral } \\
\hline RML & RLL & $\underset{\text { RLL }}{\text { RML and }}$ & LLL & $\begin{array}{l}\text { LLL and } \\
\text { Lingula }\end{array}$ & $\underset{\text { LLL }}{\text { RMLL }}$ & $\underset{\text { Lingula }}{\text { RLL, LLL }}$ & $\begin{array}{l}\text { RLL and } \\
\text { Lingula }\end{array}$ & $\begin{array}{l}\text { RUL } \\
\text { LLL }\end{array}$ & $\begin{array}{c}\text { RML and } \\
\text { LLL }\end{array}$ \\
\hline 1 & 5 & 10 & 8 & 3 & 2 & 2 & 4 & 1 & 1 \\
\hline
\end{tabular}


equivocally demonstrated in all patients, the lesion being unilateral in 27 and bilateral in 10 . The right lung was involved alone in 16, the left alone in 11 . Table 1 gives details of the lobar distribution.

Bacteriology. The sputum of 34 of the 37 patients was cultured. Table 2 gives the results.

TABLE 2

BACTERIOLOGY OF SPUTUM OF 34 PATIENTS WITH SUBACUTE PYOGENIC COLLAPSE

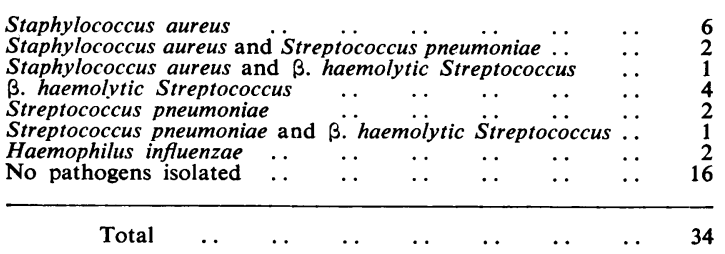

Bronchoscopy and Bronchogram. At bronchoscopic examination all patients had mucopus in the affected lobar bronchus but the bronchial lumen was not occluded by either mucous plug, mucosal swelling or glandular pressure. In 28 patients the affected lobar branches showed mild inflammatory changes, in three a red granular appearance, and in six it was normal. After the mucopus was aspirated by sucker, more was expelled from the peripheral part of the bronchial tree when the patient was made to cough by lightening the anaesthesia.

Radio-opaque oil instilled into the bronchial tree demonstrated dilated crowded bronchi. The dilatation affected the middle divisions of the bronchial tree, and was saccular in seven and cylindrical in 30 . The smaller bronchi and bronchioles were not demonstrated as oil failed to enter these structures (see Cases 1, 2, 3).

Treatment. Two principles of treatment were followed in these patients. The first was chemotherapy to promote resolution of the infection, the second postural coughing and physiotherapy to clear the bronchial tree of secretions and to aid reexpansion of the collapsed lung. Chemotherapy was limited in many patients in the early part of this study to courses of one to two weeks sulphonamide or penicillin therapy, but later one of the tetracycline group of drugs was used for periods of four to six weeks. Postural coughing and physiotherapy was carried out in most patients but in children under 3 years it is extremely difficult if not impossible to get them to cough voluntarily or carry out any breathing exercises.

Course of Disease. Fig. 2 illustrates the varied course that the disease followed. In 16 patients resolution was complete, while seven were left with permanent collapse and bronchiectasis. Partial resolution occurred in 14 patients leaving varying degrees of patchy bronchiectasis and collapse. Those patients in whom complete resolution occurred were usually diagnosed early, were usually of an age when they could co-operate with physiotherapy and were usually given adequate chemotherapy. In contrast those with failed or partially failed resolution were frequently diagnosed later, were younger and treatment was not so effectively administered.

All patients with complete resolution have remained well during the average follow-up period of six years. Of the seven patients with failed resolution, three had persistent cough and sputum and were cured by lobectomy, while the remaining four became symptom free one to two years after the initial infection and have dry bronchiectasis. Ten of the 14 patients with incomplete resolution have remained symptom free and in good health, while the remaining four temporarily developed some cough and sputum following a respiratory infection. Only one of the patients developed wax keratosis.

\section{Case Reports}

Case 1: Example of Complete Resolution. S.H. was a normal healthy girl until she attended school at the age of 5 years in February, 1951. Following a series of colds she was left with persistent cough and became pale and languid and refused to eat. When examined at the age of $5 \frac{1}{2}$ years she was thin, pale and looked ill. There was impaired percussion note, diminished air entry and crepitations over the left lower lobe and a chest radiograph showed collapse of the left lower lobe. Postural coughing produced five or six blobs of yellow mucopus, culture of which grew $\beta$. haemolytic Streptococci. Bronchoscopic examination showed mucopus in the left lower lobe bronchus, the mucosa of which was slightly inflamed but the lumen was patent. A bronchogram demonstrated collapse and cylindrical bronchial dilatation of the three basic segments of the left lower lobe (Fig. 3a).

Following treatment with penicillin, physiotherapy and postural coughing, she gradually lost her cough and sputum and in eight weeks had regained her normal health and weight. Clinically and radiologically the signs of collapse of the left lower lobe cleared. Bronchoscopy and bronchogram 12 weeks later confirmed re-expansion of the lobe and demonstrated a normal bronchial tree (Fig. 3b). The child has been observed for 6 years and has remained well and symptom free. 
\& Bronchiectasis

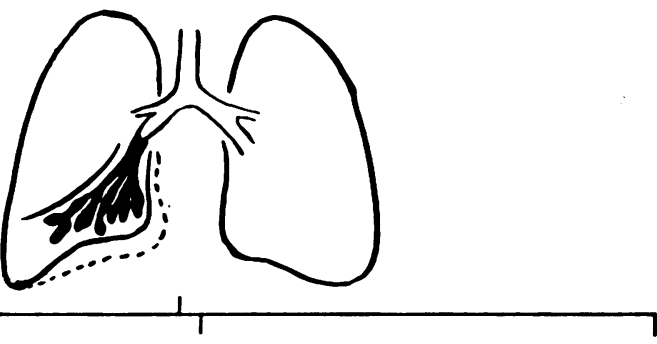

Complete Resolution

Partial Resolution

Failed Resolution

Reversible Bronchiectosis

Patchy Bronchiectasis

Permonent Bronchiectasis
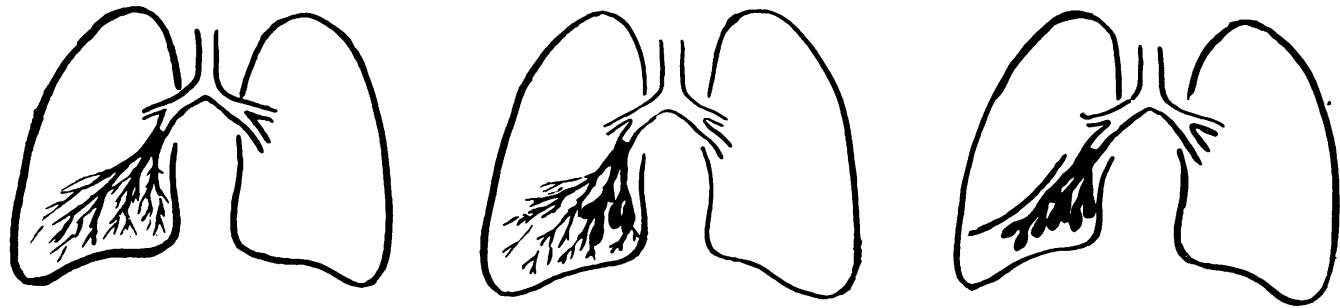

Fig. 2.-Diagrammatic representation of course of subacute pyogenic pulmonary collapse.

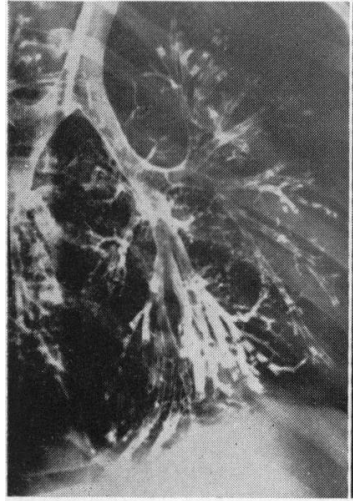

(a)

(b)

Fig. 3A.-Case 1, aged $5 \frac{1}{2}$ years. Showing collapse and cylindrical dilatation of three basic segments of left lower lobe following respiratory tract infection two months previously.

Fig. 3B.-Showing re-expansion of collapsed lobe and return of bronchial tree to normal. Bronchogram 12 weeks after Fig. 3A.

Case 2: Example of Incomplete Resolution. In February, 1950, M.P., a healthy boy of 6 years, developed an acute respiratory tract infection which was diagnosed as 'pneumonia' by his family doctor. Temporary improve- ment followed one week's sulphonamide therapy but his cough persisted and two weeks later the 'pneumonia' relapsed. Despite treatment with penicillin he remained sick and had a persistent cough. When admitted to hospital seven weeks after the original infection he appeared pale and listless and had clinical and radiological evidence of collapse of the right lower and middle lobes. Postural coughing produced blobs of yellow mucopus, culture of which failed to detect the presence of pathogenic organisms. At bronchoscopy mucopus was found in the right lower lobe bronchus but the bronchogram showed collapse, and saccular and cylindrical bronchiectasis of the right middle and lower lobes, except the axillary basic segment (Fig. 4a).

Following treatment with penicillin, physiotherapy and postural coughing, his general condition steadily improved and his cough and sputum cleared in two months. A bronchogram three months later was normal except for some slight fusiform dilatation of the more proximal bronchial divisions of the dorsal and posterior basic segments (Fig. 4b). He has been observed during the past seven years and has remained in excellent health with no cough.

Case 3: Example of Slow Incomplete Resolution. R.B., aged $2 \frac{1}{2}$ years, contracted a respiratory tract infection in November, 1949, for which he was given 


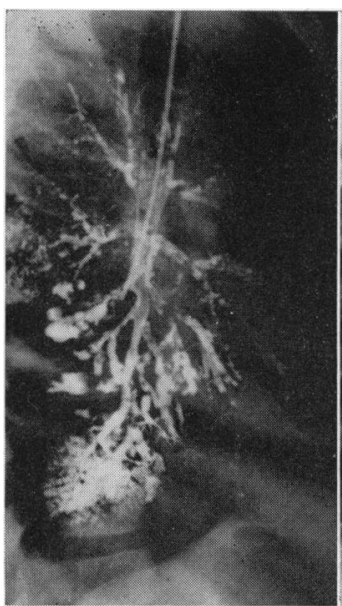

(a)

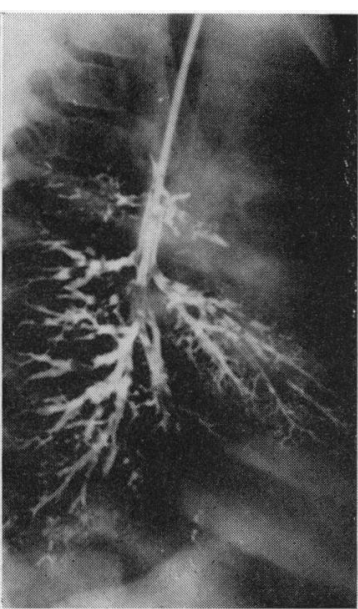

(b)
Fig. 4A.-Case 2, aged 6 years. Pneumonia seven weeks previously. Showing collapse and cylindrical dilatation of right middle lobe and saccular and cylindrical dilatation of all segments of right lobe with exception of axillary basic segment.

FIG. 4B.-Bronchogram three months after Fig. 4A, showing expansion of right middle lower lobes and a little residual fusiform dilatation of bronchi of dorsal and posterior basic segments.

penicillin for six days. Following this illness the child remained listless and pale, with low grade fever and a cough. When admitted to hospital five weeks later he had both clinical and radiological evidence of collapse of the right lower and middle lobes. On postural coughing he produced blobs of sputum, culture of which failed to detect pathogenic organisms. Bronchoscopic examination showed the mucous membrane of the right bronchus to be reddened, swollen and granular in appearance and the lumen contained mucopus. A bronchogram (Fig. 5a) showed collapse with saccular and cylindrical bronchiectasis of the right lower and middle lobes.

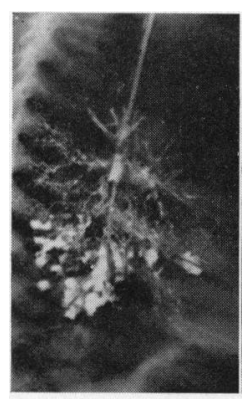

(a)

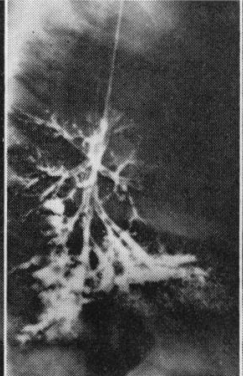

(b)

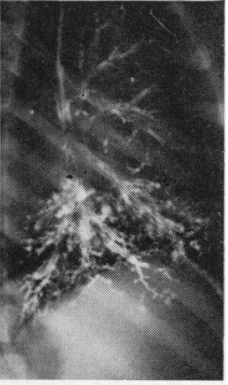

(c)
FIG. 5A.-Case 3, aged $2 \frac{1}{2}$ years. Respiratory tract infection, cough and malaise for $5 \frac{1}{2}$ weeks. Bronchogram showing collapse and cylindrical and saccular bronchial dilatation of right lower and middle lobes.

Fig. 5B.-Three months after Fig. 5A. Only change is slight reexpansion of right middle lobe with some alveolar filling.

Fig. 5C.-One year after Fig. 5B. Showing considerable re-expansion of right lower and middle lobes with residual fusiform dilatation of most of bronchi.
Treatment with penicillin and sulphonamide was given for only two weeks with slight improvement in his cough, but he was still not well and the right lower and middle lobes remained collapsed. He was too young to co-operate with physiotherapy and postural coughing. Bronchoscopic examination three months later showed a little mucopus in the right bronchus but the redness and oedema had cleared. A bronchogram (Fig. 5b) showed the right lobe was still collapsed and bronchiectatic but the right middle lobe had partially expanded, although bronchiectasis was still present.

The child slowly improved in general health and radiologically the opacity in the right lower and middle lobes gradually cleared. Another bronchogram one year later showed that the right lower and middle lobe had partially expanded and that patchy fusiform bronchiectasis involving the more proximal bronchial divisions was present in most segments (Fig. 5c).

During the following eight years he has gradually improved and over the past four years has been in excellent health. If he contracts a cold he may develop a little cough and sputum for one to two weeks.

\section{Non-Specific Infective Bronchiolitis and/or Interstitial} Pneumonia

Generalized, Patchy and Progressive Bronchiectatic Changes. Many workers among whom are McNeil, Macgregor and Alexander (1929), Robinson (1933), Ogilvie (1941), Churchill (1949), and Whitwell (1952) have shown that destructive mural bronchitis and bronchiolitis and interstitial pneumonia cause bronchiectasis and that pulmonary collapse is not an essential feature. In the following group of 57 patients it will be shown how chronic non-specific infective bronchiolitis and/or interstitial pneumonia may give rise to either generalized, patchy or progressive bronchiectatic changes and that pulmonary collapse, while it may occur, is not an essential pathological feature.

Material and Methods. Fifty-seven patients with bronchiectasis in whom the lesion commenced clinically as non-specific infective bronchiolitis and/or interstitial pneumonia were observed from the inception of the disease. All patients had negative Mantoux tests and none had fibrocystic disease of the pancreas. Methods of study were the same as in the patients with subacute pyogenic collapse.

Age, Sex Incidence, Predisposing Factors. Fortynine of the 57 children were under the age of 2 years when the disease commenced; 31 were under 1 year, 18 between 1 and 2 years and five between 2 and 3 years, and only three were over the age of 3 years. Thirty-one of the patients were girls and 26 were boys. 
These infants appeared to be in good health before the onset of respiratory infection. Eight of them exhibited manifestations of allergy either before or after the onset of the respiratory infection. A familial history of chronic sinus infection, chronic bronchitis and bronchiectasis was a feature of this group. Four siblings, one parent and three aunts had proven bronchiectasis of a similar pattern. A history of chronic cough with sputum suggestive of bronchiectasis or chronic bronchitis was obtained in 10 of the parents, in nine uncles or aunts, and three cousins.

Onset and Symptoms. In 42 of the 57 patients the disease commenced insidiously with a running nose and cough following either teething, a cold or bronchitis. In five it developed during pink disease. In the remaining 15 a definite illness, such as morbilli, pertussis or bronchopneumonia initiated the symptoms.

Persistent cough, continuous or intermittent nasal discharge, sudden attacks of fever, and general ill health were the dominant features in all patients. Ill health was manifested by lack of energy, fretfulness, irritability, poor appetite and slow weight gain. The cough often sounded as if secretion was present in the bronchial tree and in the more severely affected patients the breathing was often rattly and wheezy in character. Nasal discharge was often mucopurulent but at times mucoid in character. Febrile episodes, which were often mistaken for attacks of pneumonia, were very characteristic and occurred every few weeks or months. The onset was usually quite sudden and the attack would often rapidly abate after several days but in some persisted for longer.

Clinical Signs. Many of these children looked constitutionally ill, were often pale, undersized and had poor muscle tone and posture. Thoracic kyphosis, bilateral Harrison's sulcus and pot belly were frequently noted. Respiratory movement was poor and was mainly in the upper part of the thoracic cage. Mucopurulent or mucoid nasal discharge, with an obstructed nasal airway and oedematous red or pale mucous membrane was observed in most patients. Crepitations were the characteristic clinical sign, and in the severely affected patients were heard all over the chest, while in the others they were heard over a wider area than the lesions demonstrated both radiologically and bronchographically. Rhonchi were often heard in the severely affected patients. Thirty-six patients developed wax keratosis.
Bacteriology. It was difficult to obtain sputum from these infants and the only completely reliable way was by bronchoscopic aspiration. Irritation of the back of the throat by a spatula would result in coughing in some infants and if sputum was coughed into the pharynx it was aspirated. Sputum cultures were carried out in seven infants by pharyngeal aspiration and in 22 by bronchoscopic aspiration. In only one was a pathogenic organism, Staphylococcus aureus, cultured. However, it must be pointed out that the sputum obtained by bronchoscopic aspiration was contaminated by ether vapour and this could have been a reason for failure to culture gram negative organisms. Later these 57 children learned to expectorate mucopus and on culture the sputum in $51 \%$ grew a pathogenic organism, either $\beta$. haemolytic Streptococcus, Streptococcus pneumoniae, haemophilus influenzae, Staphylococcus aureus or bacillus proteus.

Radiological Signs. In many patients whose illness commenced insidiously plain radiographs were either normal or showed only a prominent broncho-vascular pattern. In some, particularly those in whom the disease followed bronchopneumonia, morbilli or pertussis, haziness or mottling was observed in either the lower lobes, right middle lobe or lingula. Lobar or segmental collapse was present in only 10 patients when first examined, but it developed in nine other patients later. The lingula and right middle lobe were the lobes most frequently affected.

Bronchoscopy and Bronchogram. Mucopus was aspirated from the affected lobar or segmental bronchi and only mild inflammatory changes were noted in the bronchi. In those severely affected the bronchiectatic lesions involved both upper and lower lobes. In those less severely affected, gravity seemed to play a part in determining the distribution of the lesions, for the dependent lobes namely the lower lobes, right middle lobes and lingula were affected. The lesions were commonly bilateral with the left side usually more affected than the right, and if the lesion was unilateral, it was usually the left side. The early bronchiectatic lesions usually affected the more peripheral part of the bronchial tree (Case 5)

Course of Disease. These patients were arbitrarily divided into three sub-groups according to the severity of the illness and the pattern of bronchiectatic changes. Fig. 6 illustrates these changes. The most severely affected had generalized bronchiectatic changes which were either fusiform, cylind- 
Non-Specific Infective Bronchiolitis

\& Interstitial Pneumonia

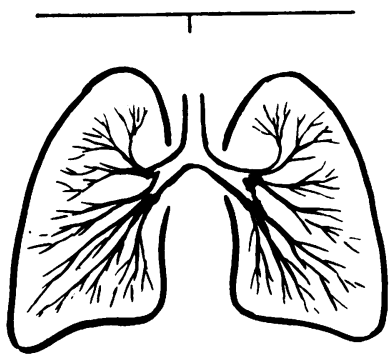

Generalised Bronchiectasis

Potchy Bronchiectasis

Progressive Bronchiectasis
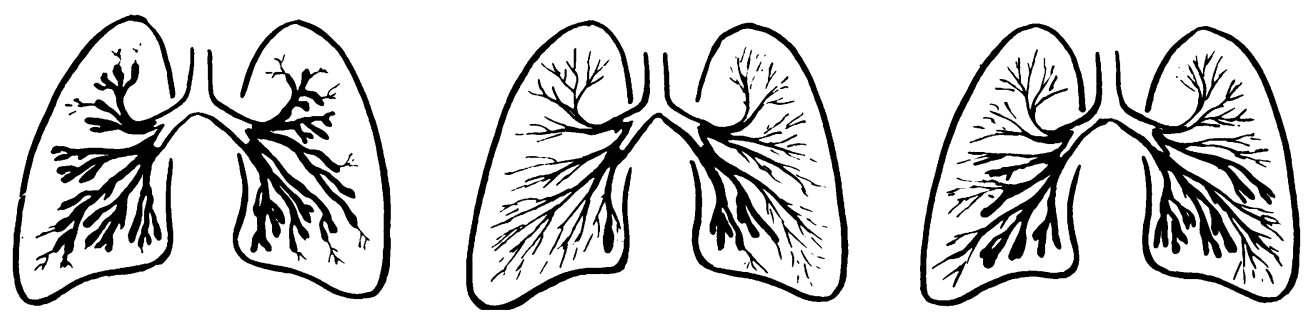

FIG. 6.-Diagrammatic representation of varied lesions that may occur in non-specific infective bronchiolitis and interstitial pneumonia.

rical or saccular in type. These children were constitutionally ill and their growth was stunted. As they grew older and could co-operate in clearing their bronchial tree of secretions, their general health improved and feverish attacks abated.

A group of 19 patients, in whom the symptoms were much less severe, had bronchiectatic changes which were either localized or patchy in distribution. In 12 the lesions were bilateral and in seven unilateral. As these patients grew older the symptoms of cough and nasal discharge ameliorated and two of them became symptomless apart from temporary cough and sputum following a respiratory infection.

In a group of 27 patients bronchiectatic changes were observed to develop in a lobe or segment previously demonstrated as normal bronchographically. Clinically this lung was affected early, as crepitations were constantly present long before bronchiectatic changes were demonstrated. It is emphasized that progression did not refer to extension of bronchiectatic changes in a segment already so affected. The most frequent pattern of development of the lesions was initial involvement of the left lower lobe and lingula and then the right middle and right lower lobes. All combinations of progressive changes have been observed in both lower lobes, lingula and right middle lobe. In 17 patients a new lobe was involved and in eight it was on the opposite side. In 10 patients a new segment was involved in an already affected lobe. Seven of the 27 patients underwent lobectomy of either one or two lobes in an attempt to remove all bronchiectatic areas. All seven patients developed bronchiectasis in another lobe, and in only two was it attributable to post-operative collapse.

Case 4: Example of Severe Generalized Type. G.L., $\frac{D}{O}$ was a normal baby of healthy parents but his maternal uncle had proven bronchiectasis. In 1954 his mother N developed non-tuberculous pleurisy, and one week later at the age of 4 months he developed a cough, rattling $\tilde{O}$ breathing and nasal discharge, and was considered to $\underset{\omega}{\mathcal{E}}$ have bronchitis. These symptoms persisted and at the $O$ age of 7 months he was found to have crepitations and inspiratory and expiratory rhonchi over the entire chest. Sputum, which was coughed into the mouth, was $\stackrel{\mathbb{Q}}{\rightarrow}$ examined bacteriologically on two occasions but no ? pathogenic organisms were cultured. Prominent broncho-vascular markings with emphysema of the lung fields 
were seen in the plain radiographs. Bronchoscopy at the age of $\mathbf{1 0}$ months showed much mucopus in the bronchial tree and bronchograms showed extensive fusiform bronchiectatic changes in the entire right lung and the major part of the left lung (Fig. 7).

Empirical treatment with one of the tetracycline group of drugs in the dose of $15 \mathrm{mg}$. per $\mathrm{kg}$. of body weight was given intermittently over the next four years. During the first two years his general health was not good and he had frequent feverish attacks and his breathing would often be rattly. However, for the past year his general health has been much better and he has not had more than two minor feverish attacks. This change has been due to his ability to cough and clear his bronchial tree of secretion.
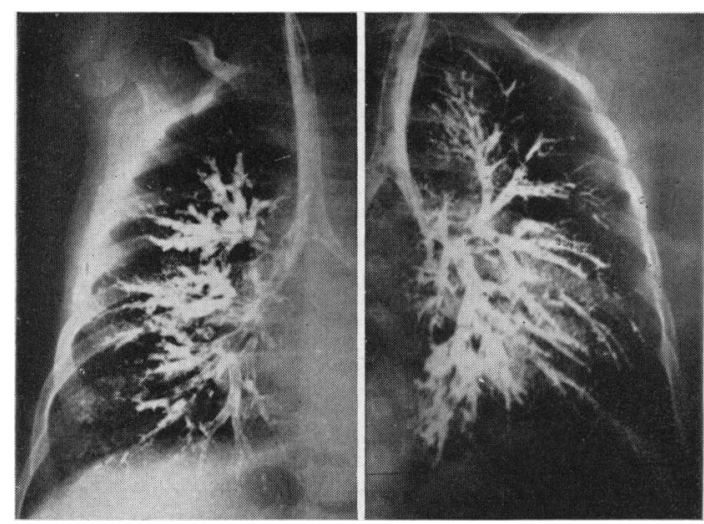

Fig. 7.-Case 4, aged 10 months. History of six months' cough, rattling breathing, feverish attacks and nasal discharge. Bronchogram showing extensive fusiform bronchiectatic changes in right lung and in left lower lobe, lingula and pectoral segments of left upper lobe.

Case 5: Example of Progressive Group. W.S. was the third child of healthy parents but a maternal uncle was considered to have bronchiectasis. When 15 months of age he developed a mild respiratory tract infection and was left with persistent cough and purulent nasal discharge. When examined in 1948, six weeks after the initial infection, he was a thin infant with purulent rhinorrhoea, slight inspiratory and expiratory wheeze and widespread fine crepitations over both lower lobes, right middle lobe and lingula. Radiological examination of his chest showed prominent hilar shadows and bronchovascular markings in both lower lobes. The cough, nasal discharge and febrile episodes persisted. At the age of $3 \frac{1}{2}$ years a bronchogram revealed a normally filled bronchial tree, except for slight stumping of one small bronchus in the posterior basic segment of the left lower lobe (Fig. 8a). A further bronchogram at the age of $6 \frac{1}{2}$ years, however, showed patchy irregular cylindrical bronchiectasis of all segments of left lower lobe, lingula and right middle lobes and of the axillary basic segment of right lower lobe (Fig. 8b).

From the age of $6 \frac{1}{2}$ years to 11 years his general health slowly improved, he lost his attacks of fever and his cough and nasal discharge lessened. At this time only a few crepitations could be heard over both lower lobes after coughing and he was able to expectorate only an occasional piece of mucopus. A bronchogram at the age of 11 years showed more pronounced bronchiectatic changes in the affected areas and in addition the retrocardiac and posterior basic segments of right lower lobe were affected.

Case 6: Example of Progressive Bronchiectasis following Surgery. M.W. was the first child of healthy parents. No familial history of bronchiectasis or other chronic respiratory illness was elicited. When aged 2 months she insidiously developed a running nose, rattling chesty

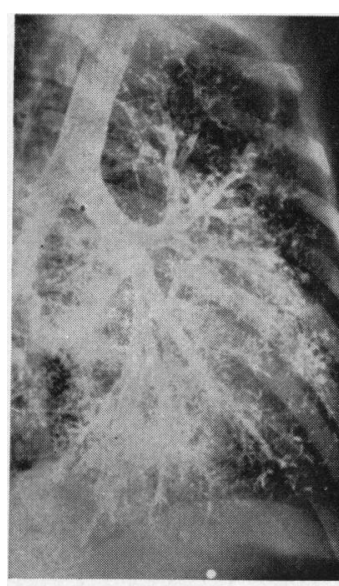

(a)

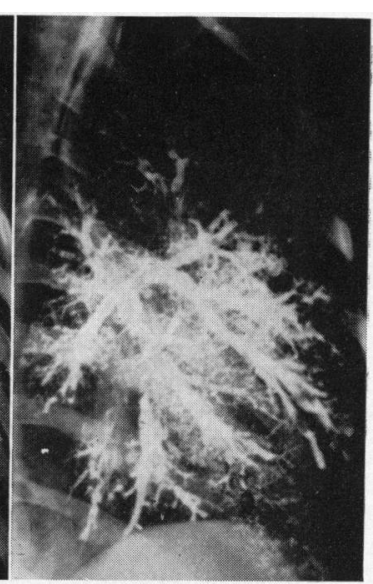

(b)
Fig. 8A.-Case 5, aged $3 \frac{1}{2}$ years. Cough, nasal discharge and episodes of fever since age of 15 months. Bronchogram showing normal filling with exception of one small bronchus in posterior basal segment.

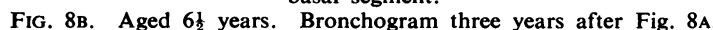
Showing patchy cylindrical lesions in all segments of left lower lobe and lingula. No collapse.

cough and later suffered with frequent feverish attacks. When first seen at the age of 8 months in 1948, she was underweight, did not look well and crepitations were heard over both lower lobes and lingula. Radiological examination of the chest showed a little haziness over the left lower lobe and the broncho-vascular lung markings were rather accentuated. The symptoms, signs and radiological changes persisted and a bronchogram at 3 years of age showed patchy irregular cylindrical bronchiectasis of the three basic segments of the left lower lobe, but the lingula and entire right lung were normal. These bronchographic changes were the same at 5 years of age.

When aged 6 years a left lower lobe lobectomy was performed. No post-operative collapse occurred but the cough, nasal discharge and febrile episodes persisted unabated. One year later a further bronchogram showed well marked bronchiectatic changes in the lingula and pectoral segments of left upper lobe, the right lung being apparently normal. Owing to persistent symptoms and 
ill health a left upper lobectomy was carried out at the age of $7 \frac{1}{2}$ years. Again no benefit followed and her general health remained poor and her sputum became profuse and offensive. Steadily inflammatory changes progressed in the right lung and her general health deteriorated, death following two and a half years later at the age of 10 years. Autopsy revealed extensive bronchiectatic changes with interstitial pneumonia in all segments of the right lower lobe and right middle lobe and less marked changes in the right upper lobe.

\section{Discussion}

Comparison of the two groups of patients, those with subacute pyogenic collapse and those with non-specific infective bronchiolitis and/or interstitial pneumonia reveal considerable differences in the pathology, pathogenesis, clinical features and course of the illness. Pulmonary collapse was the constant initial basic pulmonary lesion in the former, while it was an infrequent and incidental finding in the latter group. In the collapse group, bronchial dilatation was always present initially, and as the lesions were often reversible it is improbable that the dilatation was due to bronchial damage in many patients. In the bronchiolitis and interstitial pneumonia group, bronchiectatic lesions were not always present initially but often developed as the lesion clinically progressed. Once the bronchiectatic lesions were present they remained and reversible changes were never observed. The pattern of many of these lesions was similar to that in fibrocystic disease of the pancreas, in which widespread bronchiectatic lesions often developed from an extensive suppurating bronchiolitis. In fibrocystic disease the viscid tenacious mucus is an important factor in determining the generalized nature of the lesions, whereas gravity with poorer drainage of the dependent lobes and a poor cough reflex were important factors in the latter group. The group of patients with bronchiolitis corresponds to the patients with follicular bronchiectasis reported by Whitwell (1952), who showed that destructive mural bronchitis and bronchiolitis and interstitial pneumonia was a common cause of bronchiectasis and that pulmonary collapse was not an essential feature.

Comparison of the clinical features and course of the disease in both groups is best set out in table form (Table 3). This clinical evidence strongly supports the view that these are two different respiratory tract diseases.

TABLE 3

CLINICAL FEATURES AND COURSE OF DISEASE IN TWO GROUPS

\begin{tabular}{|c|c|c|c|c|c|c|}
\hline \multicolumn{5}{|c|}{ Clinical Feature } & \multirow{2}{*}{$\begin{array}{c}\text { Subacute Pyogenic Collapse } \\
\text { Occurs at all ages but commonest between 2-6 yr. }\end{array}$} & \multirow{2}{*}{$\begin{array}{l}\text { Non-Specific Infective Bronchiolitis and Inter- } \\
\text { stitial Pneumonia }\end{array}$} \\
\hline Age incidence & . & $\cdots$ & $\ldots$ & $\cdots$ & & \\
\hline Type of onset & $\cdots$ & $\cdots$ & $\cdots$ & $\cdots$ & $\begin{array}{l}\text { Acute with either pneumonia, non-specific } \\
\text { respiratory infection, pertussis or morbilli }\end{array}$ & $\begin{array}{l}\text { Insidious with bronchiolitis in approx. } 75 \% \text {. } \\
\text { Acute with bronchopneumonia, pertussis or } \\
\text { morbilli in approx. } 25 \%\end{array}$ \\
\hline \multicolumn{5}{|c|}{ Chronic upper respiratory infection .. } & Rarely present & $\begin{array}{l}\text { Always present: usually persistent, otherwise } \\
\text { recurrent }\end{array}$ \\
\hline Wax keratosis & $\cdots$ & $\cdots$ & $\cdots$ & $\cdots$ & Rare & Common: present in two-thirds \\
\hline Physical signs & $\cdots$ & $\cdots$ & $\cdots$ & $\cdots$ & $\begin{array}{l}\text { Always localized to affected lobe or lobes, and } \\
\text { often unilateral and unilobar. Usually crepita- } \\
\text { tions and rales, and diminished breath sounds }\end{array}$ & $\begin{array}{l}\text { Initially widespread bilateral crepitations } \\
\text { especially in lower half of chest. Clinical signs } \\
\text { more extensive than bronchographic evidence of } \\
\text { the disease }\end{array}$ \\
\hline Clinical course & & & & & $\begin{array}{l}\text { Frequently become dry and symptomless } 1-2 \text { yr. } \\
\text { after initial infection }\end{array}$ & $\begin{array}{l}\text { Nasal discharge, cough, sputum and febrile } \\
\text { episodes persist through childhood, but often } \\
\text { between ages of } 10-14 \mathrm{yr} \text {. symptoms become less } \\
\text { severe }\end{array}$ \\
\hline Radiological & $\cdots$ & $\cdots$ & $\cdots$ & $\cdots$ & Collapse always initially present & $\begin{array}{l}\text { Early: frequently little or no evidence of parenchy- } \\
\text { matous disease, especially in those with insidious } \\
\text { onset } \\
\text { Later: mottling, haziness and collapse may occur }\end{array}$ \\
\hline Bronchiectasis & $\cdots$ & $\cdots$ & $\cdots$ & $\cdots$ & $\begin{array}{l}\text { Frequently unilateral, and right side more com- } \\
\text { monly involved than left. Dilated bronchi } \\
\text { usually those of the more proximal divisions of } \\
\text { the bronchial tree. Often reversible, never } \\
\text { generalized or progressive }\end{array}$ & $\begin{array}{l}\text { Commonly bilateral and left side more exten- } \\
\text { sively involved than right. Initially dilated } \\
\text { bronchi usually those of peripheral parts of } \\
\text { bronchial tree. Lesions may be generalized, } \\
\text { localized or progressive. Never reversible }\end{array}$ \\
\hline Bacteriology & $\cdots$ & $\cdots$ & $\cdots$ & $\cdots$ & $\begin{array}{l}\text { Pathogenic organisms isolated in early stages of } \\
\text { over } 50 \% \text { cases }\end{array}$ & $\begin{array}{l}\text { No specific pathogens isolated in early stages in } \\
\text { in majority }\end{array}$ \\
\hline Genetic & $\cdots$ & $\cdots$ & $\cdots$ & $\cdots$ & $\begin{array}{l}\text { No familial history of bronchiectasis or chronic } \\
\text { respiratory disease }\end{array}$ & $\begin{array}{l}\text { Familial history of proven bronchiectasis in } 14 \% \\
\text { and of chronic sinusitis, bronchitis and probable } \\
\text { bronchiectasis in } 38 \% \text { of siblings, parents and } \\
\text { immediate relatives }\end{array}$ \\
\hline
\end{tabular}




\section{Treatment}

Three principles were the basis of the treatment of the majority of these patients. The first principle was early diagnosis to forestall some of the destructive inflammatory changes, the second was chemotherapy to assist resolution of the infection, and the third was physiotherapy and postural coughing to restore lung function and clear the bronchial tree of secretions.

In the collapse group very satisfactory results occurred in those patients in whom an early diagnosis was made, and effective chemotherapy, physiotherapy and postural coughing instituted. In the bronchiolitis group, the results were not nearly as satisfactory for two reasons. First, chemotherapy had to be used empirically as a pathogenic organism could not be isolated in the majority of patients. One of the tetracyclines in doses of $15-20 \mathrm{mg}$. per $\mathrm{kg}$. of body weight has seemed to be the most satisfactory drug. Second, difficulty was experienced with postural coughing and physiotherapy as the majority of children were under the age of 3 years. There is little doubt that inability to restore lung function was the factor of major importance in hindering the recovery of these children. It was very evident that when they were old enough to learn to clear their bronchial tree of secretions, there was very great clinical improvement and the course of the disease was in many cases arrested. Later active physiotherapy and games helped greatly to improve both general health and respiratory function.

Surgical treatment of those patients with collapse and bronchiectasis who had persistent symptoms gave excellent results. However, seven children of the bronchiolitis group who had a lobectomy subsequently developed bronchiectatic lesions in lobes previously demonstrated to be normal bronchographically. Ginsberg, Cooley, Olsen and Kirklin (1955) and others have reported similar unsatisfactory results in some children. The three premises on which the surgical treatment of bronchiectasis is based, namely that the disease is a focal one and not a general infection of the lung, second that it is not a progressive disease, and third, that a bronchogram will delineate diseased and healthy lung tissue, are not valid in this latter group. Firstly, there is good evidence to believe that the disease in some patients is a general infection of the upper and lower respiratory tract, secondly the lesions are progressive in some patients, and thirdly a lobe may be clinically infected long before there is bronchographic evidence of disease in that lobe. Moore, Kobernick and Wigglesworth (1949) showed that lung segments considered normal bronchographically showed evidence of inflammatory changes in the bronchioles. It is considered that the role of surgical resection in the bronchiolitis interstitial pneumonia group of patients should be limited to those patients who have a lobe completely destroyed by infection or by collapse and infection and who have much sputum and cough: removal of such a lobe is best deferred until the second decade when the child can co-operate with physiotherapy and when any infective lesions in other parts of the lung have been observed to be stable and not progressive.

\section{Summary}

Two groups of patients, one with subacute pyogenic pulmonary collapse, the other with nonspecific infective bronchiolitis and/or interstitial pneumonia have been shown to develop bronchiectasis.

The pathology, pathogenesis, clinical features and course, bacteriology and familial history of the two groups are different.

Treatment by chemotherapy, postural coughing and physiotherapy resulted in complete or partial resolution of the collapse and bronchiectasis in the many patients with subacute collapse. Similar treatment was much less effective in the other group owing to inability to carry out effective postural coughing and physiotherapy as the majority were under 3 years of age. In addition, chemotherapy could not be used specifically as pathogenic organisms were not isolated in most patients.

Bronchiectasis should be regarded as the end stage of a number of distinct diseases. The clinician, by recognizing the early stages of those diseases which cause permanent pulmonary damage and bronchiectasis, may do much to prevent their more serious manifestations.

Anspach, W. E. (1934). Amer. J. Dis.

Bodian, M. (1952). Fibrocystic Disease of the Pancreas, p. 135. Heinemann, London.

Brennemann, J. (1943). Dis. Chest, 9, 39.

Churchill, E. D. (1949). J. thorac. Surg., 18, 279.

Coope, R. (1948). Diseases of the Chest, 2nd ed. Livingstone, Edinburgh.

Field, C. E. (1949). Pediatrics, 4, 21

Findlay, L. (1935). Arch. Dis. Childh., 10, 61.

Fleischner, F. (1940). Amer. Rev. Tuberc., 42, 297.

Ginsberg, R. L., Cooley, J. C., Olsen, A. M. and Kirklin, J. W. (1955) J. thorac. Surg., 30,331 .

Jennings, G. H. (1937). Brit. med. J., 2, 963.

Jones, E. M., Peck, W. M. and Willis, H. S. (1946). Amer. J. Dis. Child., 72, 296.

Kent, E. M.'(1942). Amer. Rev. Tuberc., 46, 524.

Laennec, R. T. H. (1819). A Treatise on the Diseases of the Chest and on Mediate Auscultation, 4th ed. Translation by $\mathrm{J}$. Forbes and on Mediate Auscultation,

Lander, F. P. Lee (1946). Thorax, 1, 198.

and Davidson, M. (1938). Brit. J. Radiol., 11, 65.

McNeil, C., Macgregor, A. R. and Alexander, W. A. (1929). Arch. Dis. Childh., 4, 170.

Moore, J. R., Kobernick, S. D. and Wiglesworth, F. W. (1949), Surg. Gynec. Obstet., 89, 145.

Ogilvie, A. G. (1941). Arch. intern. Med., 68, 395.

Robinson, W. L. (1933). Brit. J. Surg., 21, 302.

Sant'Agnese, P. A. di (1955). Dis. Chest, $27,654$.

Veeneklaas, G. M. H. (1952). Amer. J. Dis. Child., 83, 271

Warner, W. P. and Graham, D. (1933). Arch. intern. Med., 52, 888

Whitwell, F. (1952). Thorax, 7, 213.

Williams, H. and Anderson, C. (1953). Quart. J. Med., 22, 295.

Zuelzer, W. W and Newton, W. A. (1949). Pediatrics, 4, 53. 